\title{
L'engagement poétique et politique de Samian
}

Johanne Melançon, Université d'Ottawa et Université Laurentienne

Depuis le début des années 1990, avec le succès du premier album du groupe innu Kashtin (Kashtin, 1989) formé de Florent Vollant et Claude McKenzie, on peut dire que la chanson populaire autochtone s'est taillé une place au sein de la chanson québécoise. D'abord plutôt country/folk avec des nuances de rock, cette chanson se décline aujourd'hui dans plusieurs genres et sous-genres ${ }^{1}$. Le rappeur d'origine anishnabe Samian compte parmi ceux qui se sont démarqués depuis le milieu des années 2000 dans le contexte d'un essor de la production artistique des Autochtones jusqu'à leur reconnaissance au sein de la chanson québécoise avec leur propre catégorie pour l' « artiste autochtone de l'année » au gala de l'ADISQ (Association québécoise de l'industrie du disque, du spectacle et de la vidéo) à l'automne 2019.

Samian, pseudo de Samuel Tremblay, a grandi dans la réserve de Pikogan, enclavée dans la ville d'Amos, en Abitibi, dans le nord du Québec. Il a réussi à surmonter ses problèmes de toxicomanie grâce au Wapikoni mobile, un studio ambulant de formation et de création audiovisuelle des Premières Nations. Ses débuts dans le monde musical sont marqués par la réalisation d'un premier vidéoclip intitulé « Courage », honoré au Festival des peuples autochtones unis à Pau en France en 2006. «Premier rappeur à chanter dans une langue autochtone », Samian était considéré, en 2009, comme étant, « avec la chanteuse Elisapie Isaac, l'une des deux figures de proue de la nouvelle musique autochtone canadienne » (Sabourin).

Comme le note Amy J. Ransom, très peu d'études ont été menées au sujet de la culture hiphop et du rap francophone québécois ou canadien, encore moins du rap autochtone francophone ou dans une langue autochtone (12). En fait, si le rap commence à se développer au Québec vers le milieu des années 1980, il faut attendre la fin des années 1990 pour voir apparaître une deuxième vague dont fait partie le groupe Loco Locass (Lemay 55-56). Au début des années 2000, une troisième vague « qui se caractérise par la pluralité de styles de rap qu'elle produit » et par le fait « que ses membres ne proviennent plus systématiquement des grands centres urbains » (59), se développe. Le parcours artistique de Samian s'inscrit dans cette troisième vague et dans l'émergence du rap francophone «blanc» au Québec dont le premier album de Loco Locass, Manifestif (2000), constitue le point d'ancrage (Ransom 13). C'est d'ailleurs une collaboration de 
Samian avec ce groupe pour la chanson «La paix des Braves » (2007) qui lance la carrière du rappeur $^{2}$. Le succès de la chanson et du vidéoclip qui l'accompagne, s'inscrit à la fois dans le contexte politique d'un discours postcolonial et d'un mouvement de résistance et de revendication qui ne s'essouffle pas depuis la crise d'Oka ou «résistance de Kanesatake » ainsi que d'un dialogue entre le gouvernement québécois et les Cris du Québec au moment de la signature de la « Paix des Braves » en 2002.

Par ailleurs, le rap de Samian présente des affinités avec le mouvement du rap autochtone au Canada alors que « Aboriginal rap music represents a form of resistance through the ability to bring Aboriginal issues to the attention of both those that may have lived through similar experiences and those that may merely empathize » (Manzo et Potts 181). De plus, les chansons de Samian s'inscrivent dans ce qu'on appelle le « rap conscient $»^{3}$ qui « transmet un contenu de signification qu'il met en valeur, la plupart du temps un 'message' à caractère social énoncé sur un ton dénonciateur ou revendicatif » (Barrat 18). Les quatre albums du rappeur, dont les trois premiers ont été produits au moment de la Commission de vérité et de réconciliation (2007-2015), de même que sa chanson "Génocide» témoignent du rôle social que peut jouer l'artiste. Les chansons du premier album, Face à soi-même (2007), plus personnel, constituent autant de récits de soi où le rappeur se regarde en face, parle de lui-même avec intégrité et authenticité, un parcours présenté comme une véritable thérapie qui mène à la guérison et en fait un exemple. Dans Face à la musique (2010), il pose un regard réaliste et sans concession sur sa vie et celle de son peuple tout en proposant un discours de résistance par sa pratique artistique. Enfant de la terre (2014) aborde surtout la réappropriation de son identité autochtone, avec une spiritualité assumée, insistant entre autres sur le lien avec la Terre, comme le suggère le titre de l'album. En cela, il est au diapason de ce que prône Yves Sioui Durand pour qui «[1]a naissance d'une modernité artistique amérindienne passe par la constante réaffirmation, par chaque Indien, à sa manière propre, du lien essentiel avec la Terre, ce qui constitue la spiritualité première, profonde et vivante qui est la base de notre identité moderne. C'est le défi que l'artiste contemporain autochtone doit relever » (10). Enfin, dans Le messager (2019), Samian assume son rôle de porte-parole et de militant, de celui qui s'est donné une mission de résistant, pour sensibiliser, dénoncer, revendiquer avec plus de force. Il se perçoit d'ailleurs lui-même «comme un porteur de message $(\mathrm{s})^{4}$ » (Bergeras 32-33) et il « endosse volontiers le rôle de messager, ou plutôt de militant, pour les peuples des Premières Nations » (Montpetit 6-7). D’ailleurs, le rap, ce discours rythmé où 
musique, oralité et écriture sont en constante interaction, accorde une grande place aux paroles et constitue pour l'artiste un moyen privilégié pour s'exprimer, mais aussi pour dénoncer et revendiquer un changement social et/ou politique.

Ainsi, l'esthétique du rap favorise le récit, qu'il soit politique, poétique, collectif ou encore qu'il se présente comme le récit de soi, alors que le rappeur s'adresse directement à son public et l'interpelle. Plus qu'une simple expression de la résistance, le rap constitue ainsi un mode d'expression ${ }^{5}$ de soi et cette particularité du genre constitue un atout dans le processus de décolonisation, « using those awareness-enabling, storytelling capabilities of rap to apprise listeners and the artists themselves of those effects and affirming the notion of resistance to them $»$ (Manzo et Potts 176-177), car le rap de Samian s'inscrit dans le mouvement de la « décolonisation de l'art» et de la «décolonisation par l'art» (G. Sioui Durand 24). Comme le formule Guy Sioui Durand,

[o]n ne se contente plus d'une décolonisation de l'art, c'est-à-dire d'une vague reconnaissance de l'art autochtone à l'intérieur des institutions coloniales. Nous sommes entrés dans une phase nouvelle : celle de la décolonisation par l'art. Le processus de décolonisation de l'art était fondé sur la résilience, la résistance, la contestation et la critique. Le processus de décolonisation par l'art, quant à lui, vise à compléter la réinscription des peuples autochtones dans l'histoire politique, en utilisant l'art comme avant-garde. Ce processus est loin d'être arrivé à son terme. (24)

Les chansons de Samian «sont non seulement des instances de décolonisation, ce sont des outils cruciaux et nécessaires à la diffusion, la transmission et à la continuité », comme le note Sarah Henzi («Entre orature et écriture », 198) à propos de la bande dessinée et du roman graphique qui appartiennent, tout comme la chanson, à la culture populaire.

Par ailleurs, la démarche du rappeur, à la fois personnelle, collective et fictive, implique une dimension thérapeutique en ce sens qu'elle vise la guérison, la reconstruction (ou reconquête) de soi pour lui-même et pour son peuple. Comme le souligne Véronique Audet au sujet des nouvelles musiques autochtones chez les Innus, « [o]n remarque dans ces musiques l'importance $\mathrm{du}$ thème et de la portée identitaire, ainsi que leur potentiel d'action et de transformation personnelles et collectives au moyen de l'expression et de la dénonciation des problèmes sociaux » (Innu Nikamu, 5), ou si l'on veut, leur potentiel « de revitalisation culturelle et de de guérison sociale» (Diallo 12) et symbolique, la musique pouvant être considérée «comme un lieu 
d'expression fortement chargé symboliquement, agissant sur les personnes à des niveaux cognitifs et émotionnels, dans le cadre d'un cheminement thérapeutique » (Audet, Innu Nikamu, 145).

Dans sa pratique d'un «rap conscient», Samian témoigne à la fois d'un engagement poétique où il travaille les mots et d'un engagement politique où il se fait militant en embrassant la cause des Autochtones. Son cheminement le mène de la résilience, la reconnexion avec ses origines autochtones et la résistance jusqu'à la revendication pour reconstruire le lien entre les peuples.

\section{Un engagement poétique : le rappeur et ses mots}

Avec ses plumes d'aigle ${ }^{6}$, le rappeur Samian se montre digne de cet héritage reçu des Aînés et se fait parolier - et même poète -, et une dizaine de chansons mettent en scène le rappeur en train d'écrire ou soulignent l'importance des mots " pour se découvrir » et de l'écriture comme thérapie puisqu'il est « de ceux qui croient que la plume peut guérir ${ }^{7}$ ou pour résister et combattre. Presque toutes ses chansons sont écrites au « je », confirmant qu'il s'agit pour lui d'une démarche personnelle, d'une expression de soi associée à une quête et à une (re)définition de son identité en tant qu'Autochtone et en tant qu'artiste. Cet engagement de soi à travers et envers l'écriture permet la résilience, la reconnexion et la reconquête de soi (Armstrong) puisque la plume d'aigle « soulage les âmes les plus malheureuses / [...] permet de traverser les périodes orageuses / [...] Un réconfort pour les gens qui ont besoin d'être écoutés » (« La plume d'aigle », FM)

Ainsi, le rap s'avère pour Samian un moyen de poursuivre le cheminement entrepris avec l'expérience du Wapikoni mobile : «Je poursuis ma quête / Je ne suis qu'un humain qui cherche à se connaître » (Samian, « Life », Le messager $\left.{ }^{8}\right)$. Son témoignage confirme aussi qu'il a entrepris un processus de guérison, avec le « Wapikoni qui a changé [s]a vie / qui a fait de [lu]i un autre homme » («Mon évasion » [Nitocimo8in], FS). Expression d'émotions et d'états d'âme, son rap, en tant que récit de soi, est à la fois un dévoilement de fragments de vie, une confession, une demande de pardon, une prière et surtout une thérapie qui mène à la guérison, expression de sa résilience. C'est surtout dans les chansons des deux premiers albums que l'on retrouve ce thème, en particulier la chanson liminaire de Face à soi-même, où Samian se présente, en affirmant sa simplicité, son humilité, bref, son authenticité en tant qu'artiste :

c'est mes tounes, mes histoires, mes problèmes / ma façon de voir la vie pis c'est une façon de voir la tienne / il y a des trucs sur l'album que j'ai jamais dit à personne / j'me dévoile... sans faire de mal à personne / j'suis tellement simple, c'est tout ce 
que le rap me transmet / j'suis humble et vrai ! c'est tout ce que le rap me permet (« Intro » [Kaikitomakakin], Face à soi-même ${ }^{9}$ )

Ses chansons témoignent de « [s]a réalité » ${ }^{10}$ sans cacher qu'elle ressemble souvent à un «bad trip ! »(« Mon évasion » [Nitocimo8in], FS). De plus, inspiré par une démarche spirituelle, ce dévoilement permet une confession, à la fois de faire des aveux et de solliciter le pardon. Cet aspect des chansons est dominé par la foi chrétienne : « [q]uand tout va mal, comme tout le monde, je recherche Jésus » (Samian et Esmeralda, « Higher Love », Enfant de la terre ${ }^{11}$ ), affirme Samian, lecteur de la Bible, qui avoue qu'il a «fait le con » et qui sollicite le pardon, en particulier dans deux chansons du premier album, «Pardon Remix» (Pakitentamake8in), avec deux couplets chantés en algonquin, et « Pardon » (Pakitentamake8in) qui propose un texte différent ${ }^{12}$, mais avec le même refrain :

Kika kwedjimin kidji pakidendamawijian / nikikendan eki madjidotaman. Nokom nidanawendisonan / kika kwedjimin dash kidji pakidendamawishian! / (Je vous demande pardon, je sais que j'ai fait le con / Aujourd'hui je me repens et je vous demande pardon !) (FS)

La pratique du rap s'inscrit dans une démarche spirituelle et permet à Samian de s'exprimer sous forme de prière, que ce soit pour remercier ou pour dire sa peur d'être abandonné, comme dans « Lettre à Dieu ». Cette démarche est aussi thérapeutique puisqu'elle permet de se libérer :

Prisonnier de certaines choses que je ne peux pas dire / Alors j'écris, comme on crie à haute voix / Tout ce mal être, qui s'est logé au fond de moi / Chacun son histoire, on la raconte à sa façon / Plus facile pour moi de le faire par la chanson / Je t'avoue que la musique a sauvé ma vie / Je t'avoue que c'est douloureux mais j'ai grandi (« Life », $M$ ).

L'écriture (les mots) et la pratique du rap (la musique et le rythme) s'avèrent donc des outils privilégiés pour guérir ; elles permettent dès lors la résilience : «Au lieu de fuir et de me noyer dans la boisson je préfère de loin m'enivrer dans la chanson / c'est ma thérapie avant de déraper / au lieu de boxer je préfère rapper» («Précieux lendemain », $M$ ). C'est également un moyen de ne pas retomber dans la délinquance : « ma façon de m'en sortir c'est d'écrire des raps / c'est ma façon de reprendre mes sens avant que je dérape ! » (« Mon évasion » [Nitocimo8in], $F S$ ), car les mots permettent d'atténuer la souffrance morale, puisque « [s]es écrits servent de remèdes » («À cœur ouvert»,E). Curative, l'écriture de chansons permet de « vivre bien » (Audet, Innu Nikamu, 10); de plus, comme le note Leanne Simpson, « living in a good way is an incredible 
disruption of the colonial meta-narrative in and of itself» (41). C'est que « la guérison peut advenir par l'affirmation de sa culture » (Armstrong 24).

Comme le propose Jeannette Armstrong, la reconquête de soi passe donc «par le truchement de l'écriture » (24) et dans ce contexte, l'artiste dont la pratique inclut l'écriture peut avoir recours à des stratégies de réappropriation de la langue qui, selon Henzi constitue « un outil visant une décolonisation transformative» («Stratégies de réappropriation », 76). Cette réappropriation constitue alors non seulement un « processus de récupération » mais également un « acte déterminé de résistance » (78). Il ne s'agit cependant pas de la seule stratégie à laquelle l'artiste autochtone peut avoir recours ; il peut et doit aussi se réapproprier des éléments de sa culture et de sa spiritualité, comme le suggère Yves Sioui Durand : « Nous n'avons pas le choix, nous, les artistes, de nous engager dans un effort de reconstruction et de réappropriation culturelles [...] ; une reconstruction parce qu'il y a bien eu destruction, viol, folklorisation, infantilisation et appauvrissement culturel par le détournement des croyances, l'interdiction des pratiques rituelles par la religion catholique » (138).

La quête de Samian, une reconquête de son identité autochtone, le mène, dans un premier temps, à l'affirmation d'une identité « métissée » qu'il a d'abord de la difficulté à assumer : « [...] mi-Amossois mi-Anishinape ! / c'est pour ça qu'on me rejette peu importe où je mets le pied ! / je suis mêlé, viens m'aider, je suis mal pris, je l'ai mal dit / je suis un métis et pour certains c'est une maladie ! » (« Mon évasion » [Netocimo8in], FS). C'est comme si on lui demandait de faire un choix qu'il ne peut pas faire : «J'viens de 2 [sic] mondes complètement différents, / Sur une réserve indienne mais issu d'un père blanc ! / L'héritage de ma mère, mes origines algonquines, / Tellement mêlé en cours de route, sur ma propre estime ! / Suis-je Amérindien ou blanc ?» (Samian, Shauit, «Les nomades !» [Kapapamatisi8atc], FS). Pourtant, cette double identité, il arrive à l'assumer : « Je fais partie de deux peuples, donc je finirai comme l'un d'eux !» (Samian, Biz et Batlam, « La paix des braves », FS). C'est d'ailleurs un des aspects importants de son premier album très justement intitulé Face à soi-même. Cependant, dans la plupart des chansons, il revendique son identité autochtone - « Je viens d'une réserve indienne [...] ( (Mes réserves », $F M$ ) ; « Je suis un Amérindien, j'ai pas besoin qu'on me dise d'où je viens » (« Mon évasion », [Nitocimo8in], FS) - et même une identité artistique autochtone : «7e Ciel Records, il y a un rappeur autochtone » («Les miens », E). Samian se pose comme étant le représentant de «[s]a nation » («Injustice » (Panaakentamokani8i), FS), de «[s]on peuple à travers l'art» («Plan 
Nord »,E) et de toute « une jeunesse et tout un peuple oublié » (« Warrior », FM). Il scande haut et fort sa fierté de ses origines autochtones :

J'représente ma nation, ma culture, ma passion / Ma raison de vivre ! / Mes origines, ma fierté, ma zone grise ! / Faut pas oublier que j'viens de l'Abitibi / Sur une terre algonquine, on m'a appris la vie ! / [...] / Car je représente ma Nation, bref, ma génération, / Mon peuple, mes ancêtres ! Il y a pas mille façons d'être / Je suis ce que je suis je suis fier de l'être (Samian, « Injustice » [Panaakentamokani8i)], FS).

Cette affirmation d'une identité autochtone se fait aussi par la réappropriation de sa langue maternelle, surtout dans les deux premiers albums ${ }^{13}$ alors que les titres des chansons du premier album sont tous traduits en langue algonquine, que plusieurs chansons des deux premiers albums incluent des passages dans cette langue, mais plus encore dans le cinquième et plus récent album, Nikamo (2021), presque entièrement chanté en anisnabemowin ${ }^{14}$. Sur ces premiers albums, lorsque Samian chante en algonquin, «c'est pour que [s] pen peuple puisse comprendre » (« Le rap pour moi $\left.\gg^{15}, F M\right)$. En témoigne aussi la reprise de deux chansons emblématiques de la chanson autochtone soit «Ekuan Pua» de l'auteur-compositeur-interprète innu Philippe McKenzie et «Tshinanu» du groupe innu Kashtin. Les deux chansons sont interprétées avec leurs paroles originales en innu-aimun sur lesquelles Samian superpose son rap en français. Il s'agit là d'un exemple à la fois de réappropriation de la langue et de la culture, ces chansons étant devenues des « classiques », mais aussi d'une identité « métissée », bilingue. C'est que l'adaptation de ces deux « chants identitaires $»{ }^{16}$ par l'ajout d'un texte original est hautement symbolique puisque si Philippe McKenzie est «le pionnier des musiques populaires en langue innu-aimun » (RadioCanada, « Du Teweikan »), « le père de la musique moderne innue » (Nametau Innu), Kashtin, dont la chanson «Tshinanu» est considérée comme un véritable hymne, a été le groupe emblématique de la chanson populaire innue dans les années 1990 et a tracé le chemin pour plusieurs jeunes artistes autochtones. De plus, le fait que Samian interprète «Tshinanu » avec Florent Vollant établit encore plus fortement son identité autochtone, et l'inscrit même dans une filiation de la chanson autochtone. La collaboration avec des artistes autochtones comme Florent Vollant et Sakay Ottawa (pour «Ekuan Pua ») et plus récemment le choix de produire un album presque exclusivement en langues autochtones viennent souligner l'appartenance aux Premiers peuples, autre stratégie de réappropriation identitaire pour Samian.

Dans ses chansons, Samian adopte donc aussi une stratégie de réappropriation culturelle pour affirmer son identité autochtone, confirmant sa reconquête de soi et sa reconnexion non 
seulement à sa langue mais aussi à sa culture, comme le respect envers les aînés. La nouvelle génération de jeunes aura le devoir de « rendre hommage aux aînés, de les rassurer en leur disant qu'on ne va pas abandonner» et qu'« [e]n l'honneur de leur mémoire, on marchera sur leurs traces » (Samian et Kashtin, « Tshinanu », FM), chante Samian. Ce devoir, c'est le respect de leur héritage mais aussi de leur résilience, un exemple à suivre : «Une pensée pour les aînés qui malgré tout gardent le sourire / Ça me donne espoir qu'on peut guérir / Une pensée pour les anciens qui ont ouvert le chemin / Qui, au lieu de se plaindre / ont chanté ce refrain » (Samian et Philippe McKenzie, «Ekuan Pua », FM). C'est aussi honorer leurs coutumes et leurs rituels : « On chante sur les pas du Makusham avec l'odeur de la sauge pour purifier nos aînés » (Samian et Kashtin, « Tshinanu », FM).

Une autre valeur des cultures autochtones est mise en évidence dans les textes de Samian, soit le fait de vivre en harmonie avec la terre, encore une fois dans le respect de l'héritage qu'il a reçu : «Aborigène, à mes ancêtres j'ai obéi / Lié à la terre, en parfaite harmonie » (Samian et H'Sao, « Enfant de la terre », E). La chanson « Enfant de la terre », dont c'est le thème principal, propose d'ailleurs une vision du monde exprimée d'un point de vue autochtone traditionnel. Ainsi, on « a compté les lunes / [On] a su lire le ciel en guise de boussole / [...] / calculait le temps grâce au soleil / [...] / [avait] Des forêts en guise de pharmacie », incluant une culture orale qui « [t]ransmettait notre histoire de bouche à oreilles ». De plus, Samian adhère à cette vision du monde qui veut qu'« On n'hérite pas de la terre de nos parents / On emprunte à nos enfants / De toute façon, cette terre n'est pas à nous / On est à elle, on forme un tout ». Les chansons « Sur le dos d'une tortue » (avec Florent Vollant) et «La terre a des maux » où il prête sa voix à la Terre Mère en font aussi foi.

Ainsi, en plus de la résilience, le rap et ces stratégies d'appropriation permettent à celui qui $\mathrm{a}$ « un cœur d'Amérindien » la reconquête de soi par la reconnexion avec ses origines. Mais par la pratique du rap, le rappeur «mi-Amossois mi-Anishinape » appelle aussi à la résistance et à la revendication.

\section{Un engagement politique : le rappeur des maux}

« Rap conscient », la chanson que pratique Samian est personnelle en tant qu'elle est récit de soi, mais elle est aussi sociale et engagée. Sociale, elle l'est par les sujets qu'elle aborde, que ce soit l'itinérance ou l'environnement, mais principalement la situation des Autochtones victimes 
de la colonisation. En fait, l'œuvre de Samian s'inscrit tout à fait dans le mouvement du rap autochtone au Canada en abordant les thèmes de la perte du territoire et de la culture, de la « culture de la pauvreté », de la discrimination, de l'oppression, de même que de la revendication et la sensibilisation $^{17}$. De ce point de vue, sa pratique artistique est engagée parce que militante, et Samian s'affiche comme porte-parole d'une cause : il sensibilise, dénonce, résiste, invite à l'action et revendique.

Dans ses chansons, Samian endosse entièrement son rôle de porte-parole, tout comme le laisse entendre la chanson titre de son dernier album, Le messager. On peut d'ailleurs lire toute cette chanson comme un (auto-)portrait à la troisième personne de Samian en tant que rappeur qui se fait le porte-parole de sa communauté, un rôle qu'il assume d'ailleurs depuis le premier album : «J'parle pour mon peuple ! » (« Reconnaissance » [Mik8etc inentamowin], FS) et ce, de façon inconditionnelle : « je viens d'une p'tite réserve au nord [...] / je rappe pour les miens jusqu'à ma mort ! » («Intro » [Kaikitomakakin], FS). Même si la responsabilité est grande, il accepte d'être un « role model» : « On m'a donné un rôle sur du rap and roll / Je porte le fardeau d'un role model / C'est pour les miens / Les miens / «I make music for my people » (« Les miens », E).

Mais si Samian dénonce notre indifférence face à l'itinérance ou notre attitude envers l'environnement, la principale cause qu'il défend est celle des Autochtones. Par exemple, il dénonce le racisme dans «Reconnaissance» ([Mik8etc inentamowin], FS), mais aussi l'expérience des pensionnats en se mettant dans la peau de quelqu'un qui les a subis dans sa chanson « Blanc de mémoire », dont le titre suggère toute la difficulté d'oublier les traumatismes dont les Blancs ont été la cause. Cette chanson propose un récit de vie à la première personne - à six ans, seize ans, trente ans, soixante ans - un témoignage pour permettre la guérison et ce, paradoxalement, grâce à Dieu :

J'ai une boule dans la gorge quand je repense au pensionnat / [...] / On m'a violé, traité comme si je ne valais rien / J'ai 6 ans, je me sens seul et ma famille me manque / [...] / Aujourd'hui j'ai 16 ans et je n'ai jamais pleuré une seule larme de mon corps / On n'a pas le droit d'imposer à un enfant sa religion [...] / Aujourd'hui j'ai 30 ans et j'ai repris ma vie en main / On m'a présenté le même dieu, mais celui-ci était amour $/[\ldots] /$ J'arrive à 60 ans et j'ai l'impression de renaître / [...]/ Je quitterai ce monde comme j’y suis venu, libre, comme un Sauvage. («Blanc de mémoire », E)

Par ailleurs, s'il reconnaît les problèmes de toxicomanie - « on dit des autochtones, / qu'on a un problème de coke, / pis un problème de boisson / le pire c'est que t'as raison / on est pris dans un monde de consommation » (« Ma réalité » (Eici bapataman), FS) -, il ne fait pas que les constater, 
il invite à réagir : «Il est temps qu'on avance, qu'on se rassemble pour la cause / Qu'on arrête de se détruire par l'alcool et la coke » («Peuple invincible », FM).

Le discours de Samian est donc principalement inspiré par la critique du colonialisme, s'inscrivant ainsi dans un plus large courant de discours militant. En témoigne entre autres la chanson "Peuple invincible »-allusion bien sûr au documentaire choc sur les Abitibiwinni de Richard Desjardins et Robert Monderie, Le peuple invisible (2007) - dont le thème principal est justement la dénonciation du colonialisme, en particulier auprès de la nation algonquine de l'Abitibi d'où est originaire Samian: "Car pour vous dire la vérité, ils ont essayé de nous déraciner » (Samian, « Peuple invincible », FM). C'est le militant qui s'affirme : « Il est temps [...] / Qu'on leur prouve qu'on est des hommes, qu'on est fiers de qui on est / S'ils nous traitent de sauvages, on s'en fout, on est des guerriers ». Bref, il s'agit de «briser le silence, la honte et la gêne / D'un peuple invisible, comme le dirait Desjardins ». Dans « Mino Picaok (ooma kopek aki) » $(F M)$, il aborde pour une rare fois un aspect politique de la question autochtone, soit les revendications territoriales ${ }^{18}$ et il dénonce le génocide de son peuple : «Pour moi, 1604 est la plus grosse perte territoriale / On est la plus grosse extermination de l'histoire / C'est ce qu'on a oublié de te dire dans ton livre d'histoire / [...] / Il y a une loi sur les sauvages et je me dois de riposter ». Ce discours est encore plus appuyé dans sa collaboration avec le groupe rap Sans pression pour la chanson «Premières Nations » (La tendance se maintient) dont le refrain, scandant entre autres la devise du Québec « Je me souviens », vient justement souligner l'« oubli » par les Européens que le continent qu'ils ont « découvert », était déjà habité. En fait, son propos est encore plus percutant dans sa dernière chanson, «Génocide ${ }^{19}$, écrite cinq ans après le dépôt des recommandations de la CVR qui concluait à un « génocide culturel » puisque c'est « [t] oujours le même combat » :

$\mathrm{Au}$ nom des enfants vendus et déracinés / Au nom des femmes disparues et assassinées / Des femmes ligaturées à leur insu / Un Génocide bien planifié, et ça continue / [...] / L'interdiction de parler nos langues, c'est un Génocide / Tuer l'indien à l'intérieur de l'être humain, c'est un Génocide / Voler et vendre des enfants, c'est un Génocide / Les femmes autochtones disparues et assassinées, c'est un Génocide / La constitution canadienne, est un Génocide

Ainsi, Samian aborde la question autochtone non pas pour «jouer la victime» («Warrior », $F M$ ) mais pour appeler à la résistance. Son « rap conscient» incite à l'action, au combat : «Le poing dans les airs, One time for my Warriors / J'lâche pas le combat, je fonce dans le tas / Comme un soldat, ici-bas, j'ai un mandat / [...] / Quand je rappe je déclenche des débats » («Les Miens », E). Bien plus, par son « rap de résistant », Samian «prône le changement » et 
« rêve d'un coup d'État! » («Les miens ») : «J'essaie de changer l'monde avec mes cordes vocales! » («Injustice » [Panaakentamokani8i], FS). Sa pratique artistique sert donc à combattre, comme en témoigne le vocabulaire guerrier que ce «warrior $»^{20}$ des mots emploie : il est temps qu'il «passe à l'attaque » («Pardon » [Pakitentamake8in]) et « [c]'est par le son qu['il] par[t] au front pour une révolution» ( Hold the Mic », E) puisqu'il « fai[t] partie de ceux qui veulent se battre » («Mon évasion » [Nitocimo8in], FS). Il affirme même : « je suis prêt pour la guerre et je laisserai personne derrière / je vais me battre jusqu'au bout comme un militaire ». Bref, s'il « combat[s] par les mots » (« Mes idéaux », $F M$ ), c'est qu'il est « un warrior... un vrai combattant / Parce que le rap, pour [lui], c'est beaucoup plus qu'un passe-temps ». Il se réapproprie et renverse ainsi l'image du stéréotype de «l'Indien » guerrier héritée des livres d'histoire, des livres scolaires et de la culture populaire (Henzi, « Stratégies de réappropriation »).

Un autre objectif le guide, soit de redonner espoir : «C'est de l'espoir pur et dur que je grave sur un disque » (Samian, «Warrior », FM). C'est le message de sa reprise et adaptation du succès de Kashtin « Tshinanu » où il s'adresse d'abord aux jeunes Autochtones :

Faudra dire à nos enfants que le monde leur appartient, qu'on peut croire en l'impossible et qu'on n'est pas les plus à plaindre. / [...] / On n'est pas les victimes mais une autre génération. / Celle qui a le pouvoir de nous offrir un horizon, de rattraper le temps perdu, de rendre hommage aux aînés, de les rassurer en leur disant qu'on ne va pas abandonner. / Ça sera long... et les épreuves seront tenaces. / Faudra du cœur, du courage, de la force et de l'audace. / En l'honneur de leur mémoire, on marchera sur leurs traces. / La tête haute comme des braves, parce qu'ici, c'est notre place. (Samian et Kashtin, « Tshinanu », FM)

C'est aussi celui de la chanson « Délivrez les jeunes » dont il est solidaire :

On est là pour donner de l'espoir à la jeunesse / C'est pour notre génération qui se perd dans l'ivresse / C'est pour les hommes, les femmes / Les forts, les braves / C'est pour ceux qui se lèvent et non ceux qui s'écrasent / C'est ensemble qu'on vaincra, qu'il y aura une révolution / Ou du moins l'espérer pour le temps d'une chanson / On a envie de croire, dans notre musique, il y a de l'espoir / [...] / On vient briser les chaînes, on rêve de liberté / On rêve de vivre ensemble, alors, laissez-nous rêver ! $(F M)$

Pour Samian, dès la première chanson de l'album Face à soi-même, le rap constitue une forme d'art performative : «j'ai décidé de faire du rap et c'est le rap qui me travaille / [...] / sans même poser un geste on peut faire une action! » («Intro » (Kaikitomakakin)). Aussi, après la résilience, la reconnexion, la résistance et la revendication, il est temps de passer à la reconstruction (Y. Sioui Durand). 


\section{Pourquoi rapper ?}

Pourquoi chanter quand il y a tant à faire

(Luc Granger, « Pourquoi chanter »)

De Face à soi-même jusqu'à la chanson « Génocide », l'œuvre du rappeur Samian fait preuve d'une grande cohérence. Sa démarche, d'abord très personnelle, où la chanson est d'abord l'expression de soi, lui permet d'effectuer une quête d'identité sous forme de confession, d'aveu et de demande de pardon, et surtout d'accepter sa part d'héritage autochtone jusqu'à élargir son propos à la sensibilisation aux problèmes de toxicomanie et à la destruction de l'environnement, à la dénonciation des injustices, de la discrimination et du colonialisme, de même qu'à la revendication d'une reconnaissance des peuples autochtones. Une fois la part de son identité autochtone assumée, et grâce à sa façon toute personnelle d'intégrer à sa pratique artistique une spiritualité qui tient de son héritage autochtone et de son héritage chrétien, il peut appeler à la résistance et revendiquer des changements. Il devient alors le porte-parole d'un projet pour une société plus humaine et universelle.

Même s'il s'affirme comme « militant » et comme « résistant » qui prône une révolution, Samian choisit l'art plutôt que la politique. À la question d'un chroniqueur lui demandant « Qu'estce que l'art peut ajouter au discours politique? », Samian répond :

L'art, c'est rassembleur. La politique, ça divise. On m'a beaucoup approché en politique dans les dernières années. J'ai parlé avec des politiciens et je crois qu'en politique, tu n'as pas le choix de prendre la parole pour dire ce que les gens veulent entendre. J'ai vraiment cette impression-là. [Les politiciens] sont prisonniers de leur discours, ils doivent se censurer. Un artiste, non. Un artiste a sa philosophie, sa façon de penser et il rassemble beaucoup plus qu'un politicien peut le faire. (Aubert 1-2)

C'est d'ailleurs ce qu'il affirmait dès son premier album : « Le rap pour moi c'est plus fort que la politique » (« Le rap pour moi », FM).

Refonder la relation entre Autochtones et allochtones, voilà le projet du rappeur, dès le premier album, avec la chanson « La paix des braves » : « Samian, Loco Locass ! / Il était temps que l'union se fasse / Qu'on remonte la vraie histoire / Pour qu'on puisse y faire face ! / On veut mettre un pont entre les Nations » (Samian, Biz et Batlam, FS). Pour ce faire, les paroles prônent la réécriture de l'histoire de l'arrivée des Européens en Amérique pour dissiper le malentendu historique de sa « découverte » et appelle, dans son refrain bilingue, à une nouvelle alliance entre Autochtones et allochtones (francophones) : 
Hochelaga, Stadaconé ! / On débarque en rabaska / C't'année, on a décidé de se métisser / Parce qu'on a retissé l'alliance en Nouvelle-France // Entre autochtones et francophones! / Mamawintiwin mi iima eiji mackawisiak, mackawisiak / Eawiakowiak etitentakosiak! (La force vient de l'unité) / C'est en mêlant le son qu'on scelle le pacte ! »

C'est le même message qu'il faut lire dans cette allusion de celui dont le prénom de baptême est Samuel : «Appelez-moi Samian de Champlain » (« Rez », E). La substitution de la traduction en langue algonquine de son prénom (Ransom 17) est hautement symbolique, à la fois (re)prise de possession du territoire et affirmation d'une identité hybride.

L'autre aspect du projet énoncé par le rappeur est de construire un monde plus humain, audelà de la politique et des religions, car au-delà de son appartenance à la nation algonquine, Samian veut inclure l'ensemble de l'humanité dans ses revendications : «C'est vrai qu'à quelque part je revendique pour les miens / Mais t'as pas compris, je me bats pour les droits de l'être humain » (« Hold the Mic », E). Ce discours humaniste s'affirme déjà dans le second album, Face à la musique: «mais le désir de mon cœur, c’est qu'un jour on soit frères » (« Mino Picaok »). Optimiste, le rappeur « rêve de voir le monde, de haut, de là, quelque part / Au-delà des religions et au-delà de ce qui nous sépare » (Samian et Marième, « Regarde ailleurs », FM). Et en fait, il n’y a pas que la religion qui sépare : « Ceux qui séparent par la couleur, par la religion ou par la peur / Ces gens-là n'ont pas de cœur et un jour ils le sauront / Mais ceux qui rêvent d'un monde meilleur, qui acceptent les gens d'ailleurs / Rêvant d'être frères et sœurs, mais un jour, ils le seront » (« Mino Picaok », FM). Il est véritablement un « enfant de la terre » : «Mon pays n'a pas de frontière / Il n'a pas d'couleur / [...] / Mon pays n'a pas de drapeau / Car on est tous les mêmes / Derrière nos couleurs de peau / Connectés à la voix du Créateur » («Enfant de la terre », E). Cette ouverture sur le monde transparaît dans sa collaboration avec le groupe montréalais d'origine tchadienne H'Sao et avec Marième (Ndiaye) d'origine sénégalaise par son père pour la chanson « Regarde ailleurs » qui invite, justement, à découvrir les autres cultures et démontre que l'on peut « être autochtone et s'inscrire dans le monde en devenir » (Y. Sioui-Durand 156) :

Regarde ailleurs, tant de cultures à découvrir / [...] / Regarde ailleurs, on ignore tout de ce monde / [...] / Je rêve de voir ailleurs, de mettre les pieds en Afrique / De visiter chaque pays et non la guerre qui les habite / [...] / Il faudra se mettre ensemble si on veut que ça change / Qu'on puisse vivre ensemble, mais par la foi / [...] (Samian et Marième, « Regarde ailleurs », FM).

Mais par-delà de ce message de fraternité entre les peuples, l'engagement auquel Samian accorde le plus d'importance puisqu'il inclut tous les autres, c'est celui de l'authenticité de 
l'artiste dans la pratique de son métier : «Qui rêve de faire le tour du monde avec son art / Qui rêve d'être réel et non une superstar / J'aime la musique pour son histoire et son message / Mais je déteste la business où la plupart ont deux visages » (« Hold the Mic », E). Les premiers mots d'« Intro » (Kaikitomakakin) sur son premier album nous l'indiquaient déjà : « Je me présente Samian, je débarque dans le hip hop / [...] / aujourd'hui je rappe pour ma cause pis je t'en offre une dose » $(F S)$. Samian est « un exemple de réussite » (« Warrior », FM), sans qu'il ait eu à renier une part de qui il est : «Je suis l'exemple qu'on peut réussir tout en restant soi-même / Même quand on grandit sur une réserve indienne » (Samian et Esmeralda, « Higher Love », E). Chaque album présente des chansons où il est question de l'écriture, du pouvoir des mots, et c'est l'écriture qui a permis cette reconquête de soi. Résilient, il contribue, comme artiste, à « la résistance, [de] la sauvegarde et [de] la reconstruction culturelle » (Y. Sioui Durand 136) de son peuple. Il s'approprie le rap en s'y exprimant en partie, et de plus en plus, dans sa langue maternelle et en y mettant en valeur la culture des Premiers peuples pour dénoncer et revendiquer, mais aussi pour proposer « une vision d'avenir nouvelle pour l'ensemble de [son] peuple » (Armstrong 25). Il répond ainsi au souhait d'Yves Sioui Durand pour qui « [t]outes les nouvelles pratiques d'art doivent être mises à profit et jouer un rôle majeur dans la reconstruction culturelle de nos Nations afin de contrer le désespoir et d'ouvrir l'imaginaire des jeunes générations » (156). Et force est de constater qu'avec Nikamo, son dernier album, le rappeur Samian poursuit cette trajectoire engagée, cette fois-ci en soulignant encore plus son appartenance autochtone comme s'il avait accompli cette reconquête de soi, ce qui lui permet de contribuer pleinement à la reconstruction culturelle qu'appelait Yves Sioui Durand.

\section{Bibliographie}

Aubert, Robin. « Des artistes qui déstabilisent ». La Presse. 23 juin 2018, arts. 1-2.

Audet, Véronique. Innu Nikamu. L'innu chante. Pouvoir des chants, identité et guérison chez les Innus. Québec : PU Laval, 2012.

---. «Hip-hop autochtone au Québec. Oralités contemporaines au gré des ondes politiques et médiatiques ». Inter art actuel 104 (2009-2010). 34-35.

Armstrong, Jeannette. « Les Autochtones d'Amérique du Nord : dépossession et reconquête de soi par l'écriture ». Nous sommes des histoires. Réflexions sur la littérature autochtone. Marie- 
Hélène Jeannotte, Jonathan Lamy et Isabelle St-Amand, dir. Montréal : Mémoire d'encrier, 2018. 21-26.

Barrat, Julien. Le rap ou l'artisanat de la rime. Paris : Harmattan, 2008.

Bergeras, Yves. « Samian : les mots du monde ». Le Soleil. 9 septembre 2019. 32-3.

Desjardins, Richard et Robert Monderie. Le peuple invisible. L'Office national du film, 2007. En ligne : https://www.onf.ca/film/peuple invisible/

Diallo, David. «La musique rap comme forme de résistance?». Revue de recherche en civilisation américaine 1 (2009). En ligne : $\underline{\mathrm{http}}$ ://journals.openedition.org/rrca/80

Henzi, Sarah. «Stratégies de réappropriation dans les littératures des Premières nations ». Studies in Canadian Literature / Études en littérature canadienne 35.2 (2010). 76-94.

---. « Entre orature et écriture : souveraineté, décolonisation et culture populaire autochtones ». Canadian Literature 230-231 (2016) : 198-213.

Lemay, Sylvain. «Analyse des messages dans le rap francophone du Québec : entre contestations, résistance, opinions et revendications (1990-2012)». Mémoire (M.A.) Université du Québec à Montréal, 2016. En ligne : https://archipel.uqam.ca/8683/

Manzo, J. et J. J. Potts. « Rez Style : Themes of Resistance in Canadian Aboriginal Rap Music ». The Canadian Journal of Native Studies 33.1 (2013). 169-188.

Montpetit, Caroline. « Samian, sans réserve ». Le Devoir, 31 août 2019. 6-7.

Nametau Innu. «Innu Nikamu : concert de Philippe Mckenzie ». Nametau Innu. Mémoire et connaissance du Nitassinan. http://www.nametauinnu.ca/fr/culture/nation/detail/65/79

Radio-Canada. « Un génocide au compte-goutte : entrevue avec Samian ». 11 octobre 2020. https://ici.radio-canada.ca/ohdio/premiere/emissions/dessine-moi-undimanche/segments/entrevue/204068/samian-genocide-videoclip-autochtones

---. « Du teweikan à l'électro: Philippe McKenzie ». 4 janvier 2018. https://ici.radiocanada.ca/teweikanalelectro/timeline/philippe-mckenzie/

Ransom, Amy. J. «"Québec History X” : Re-Visioning the Past Through Rap ». American Review of Canadian Studies 43.1 (2013). 12-29.

Sabourin, Clément. «Pop inuit, rap algonquin, reggae innu : la musique autochtone se métisse ». La Presse, 4 octobre 2009. https://www.lapresse.ca/arts/musique/200910/04/01-908277pop-inuit-rap-algonquin-reggae-innu-la-musique-autochtone-se-metisse.php 
Simpson, Leanne. Dancing on our Turtle's Back. Stories of Nishnaabeg Re-creation, Resurgence and A New Emergence. Winnipeg: Arbeiter Ring, 2011.

Sioui Durand, Guy. «Autochtones : de la décolonisation de l'art par l'art». Liberté, « Premiers Peuples : cartographie d'une libération » 321 (2018). 24-26.

Sioui Durand, Yves. OKIHOÜEY ATISKEN - L'ESPRIT DES OS. Écrits théoriques, poétiques et polémiques. Québec : PU Laval, 2020.

\section{Discographie}

Samian, Face à soi-même, CIE2-3416, 7ième Ciel Records, 2007.

Samian, Face à la musique, CIE2-3877, 7ième Ciel Records, 2010.

Samian, Enfant de la terre, CIE2-4012, 7ième Ciel Records, 2014.

Samian, Le messager, AS-006, L’Armure du son, 2019.

Samian, « Génocide », Musique nomade, octobre 2020.

https://musiquenomade.bandcamp.com/track/g-nocide

\footnotetext{
Notes

${ }^{1}$ Pour explorer la musique et la chanson autochtones contemporaines, on pourra consulter la plateforme d'écoute de Musique nomade à l'adresse https://nikamowin.com/fr.

${ }^{2}$ La chanson atteint le « top 5 » du palmarès francophone et la première place sur Musique Plus avec le vidéoclip au cours des semaines suivant le lancement de l'album. Pour un commentaire du début de la carrière de Samian voir Véronique Audet, « Hip-hop autochtone ».

${ }^{3}$ Voir le site officiel de Samian à l'adresse https://www.samianmusique.com/

${ }^{4}$ Comme le note Véronique Audet, chez les Innus, «[1]e chanteur-musicien, kanikamusht, est souvent considéré comme un messager, katipatshimusht » (Inпu Nikamu, 215).

${ }^{5}$ Selon David Diallo, « qualifier simplement la musique rap de forme de résistance est fortement réducteur dans la mesure où ce mode d'expression rassemble sans conteste une grande variété de thèmes et de discours ».

${ }^{6}$ Symbole important pour les Autochtones, La plume d'aigle est aussi le titre du recueil dans lequel Samian a réuni le texte de plusieurs de ses chansons, paru en 2014 dans la collection « poésie » chez Mémoire d'encrier, une maison d'édition qui se consacre entre autres à la publication de textes d'écrivains des Premières Nations. Sur la quatrième de couverture, on peut lire : «Samian a reçu quatre plumes d'aigle des aînés. Fort de cet héritage, il place l'écriture au cœur de la vie amérindienne. Il rend grâce aux Ancêtres. Guerrier alimentant le feu, il écrit et chante pour que leurs voix ne soient pas trahies ».

${ }^{7}$ Samian, «La plume d'aigle », Face à la musique. Désormais, la référence aux chansons sera donnée de la façon suivante : nom de l'artiste ou des artistes ayant écrit les paroles, titre de la chanson, titre de l'album. Désormais, les références à l'album Le messager seront indiquées par le sigle FM.

${ }^{8}$ Désormais, les références à l'album Le messager seront indiquées par le sigle $M$.

${ }^{9}$ Désormais les références à l'album Face à soi-même seront indiquées par le sigle FS. Sauf indication, les paroles des chansons sont de Samian.

${ }^{10}$ « Eici 8apataman eci nakosian / Pinta inapin nimatcise8inikak / Anic ka8in nitito8in kicpin / Pikosek ki tee ! (c'est ma réalité... mon identité... viens faire un tour dans ma zone grise... / pis c'est pas moi le responsable si ton cœur se brise !) » (« Ma réalité » [Eici 8apataman], FS).

${ }^{11}$ Désormais les références à l'album Enfant de la terre seront indiquées par le sigle $E$.
} 
${ }^{12}$ Les deux versions proposent aussi une musique différente, "Pardon Remix » intégrant des voix et un « rythme battant, caractérisé par des coups forts et des coups faibles, longs et courts, se succédant [est] souvent nommé le 'tempo indien' » (Audet, Innu Nikamu, 64). On pourrait en déduire que cette version s'adresse d'abord aux Autochtones, alors que l'autre s'adresse principalement aux allochtones.

${ }^{13}$ Le troisième album n'inclut qu'une chanson interprétée en innu-aimun et en français alors que le quatrième album n'inclut aucun titre ni aucun passage dans une langue autochtone.

${ }^{14}$ Les paroles des chansons, que l'on peut consulter sur le site de l'artiste, incluent une traduction et, dans un cas, une traduction du refrain et une traduction non littérale «mais plutôt comme une trame narrative » des couplets. On y retrouve des chansons en anishinabemowin, en innu-aimun et des passages en mi'kmaq. La chanson "Génocide ", premier extrait de l'album sorti en 2020, est principalement en français avec des passages en anishinabemowin.

${ }^{15}$ Le refrain de cette chanson est d'ailleurs en langue algonquine et n'est pas traduit dans le livret accompagnant le CD.

${ }^{16}$ Voir la ligne du temps proposée sur le site de Radio-Canada, Du teweikan à l'électro: Histoire musicale des 11 nations autochtones du Québec à l'adresse https://ici.radio-canada.ca/teweikanalelectro/ligne-du-temps/

${ }^{17} \mathrm{Ce}$ sont les cinq thèmes du rap canadien de l'Alberta qu'identifient J. Manzo et J.J. Potts : « Five specific themes of resistance follow : Loss of Land and Culture, The Culture of Poverty, Discrimination, The Struggles of Aboriginal Life, and Action and Awareness ».

${ }^{18} \mathrm{On}$ peut visionner le vidéoclip réalisé à partir de cette collaboration de Samian à l'adresse https://www.youtube.com/watch?v=JZW14s65scg Cette collaboration avec Sans pression contribue aussi à associer la cause des Autochtones à celles des Noirs. Cet aspect, Samian l'avait d'ailleurs déjà abordé dans la chanson « Injustice» (Panaakentamokani8i) où il comparait la situation des Premiers Peuples à celles des Africains.

${ }^{19}$ La sortie de la chanson, d'abord prévue pour 2021, a été devancée en octobre 2020 suite aux événements entourant la mort de Joyce Echaquan (Radio-Canada, «Un génocide »). Elle est accompagnée d'un vidéoclip: https://www.youtube.com/watch?v=cYSTngC96H8\&t=7s

${ }^{20}$ Samian arbore le mot « warrior » tatoué dans son cou. 